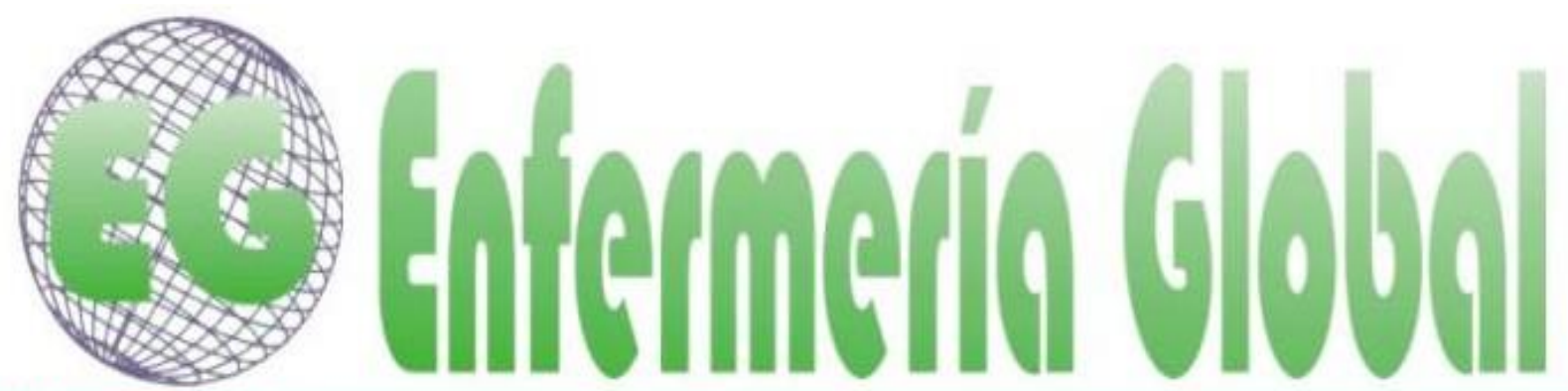

\title{
Funcionamiento y calidad de vida en personas con enfermedades crónicas: poder predictivo de distintas variables psicológicas
}

Functioning and quality of life in people with chronic diseases: predictive power of different psychological variables

\section{*Pascual Sánchez, Ana **Caballo Escribano, Cristina}

*Universidad de Salamanca. E-mail: anapascual@usal.es **Universidad de Salamanca. INICO. España.

http://dx.doi.org/10.6018/eglobal.16.2.243031

\section{RESUMEN}

Objetivos: Evaluar variables emocionales (ansiedad, depresión), cognitivas (catastrofismo, autoeficacia percibida) y conductuales (estrategias de afrontamiento); conocer de qué modo este tipo de variables influyen en la calidad de vida relacionada con la salud en personas con enfermedades crónicas; analizar si existen diferencias significativas en función del género o del diagnóstico de enfermedad crónica en las variables psicológicas mencionadas; detectar si se dan diferencias significativas en las distintas dimensiones de calidad de vida entre población normal y población con enfermedades crónicas.

Método: Estudio descriptivo-correlacional realizado en los meses de febrero y marzo de 2015 en el que participaron 35 personas con diferentes diagnósticos de enfermedad crónica, de las cuales 24 $(68.6 \%)$ eran mujeres y $11(31.4 \%)$ varones.

Resultados: Los resultados obtenidos sugieren que las variables emocionales repercuten en la calidad de vida, al igual que la autoeficacia y algunas estrategias de afrontamiento. Se encuentra además que las personas evaluadas presentan niveles de calidad de vida significativamente menores que la población general, y que parecen existir pocas diferencias en las variables evaluadas entre personas con diagnósticos diferentes y entre hombres y mujeres.

Conclusiones: Las variables psicológicas juegan un papel importante en la calidad de vida de las personas con enfermedades crónicas, por lo que es necesario abordarlas. Los resultados obtenidos son acordes a los encontrados en estudios previos, aunque es recomendable seguir investigando con muestras más amplias.

Palabras clave: enfermedad crónica; calidad de vida relacionada con la salud; autoeficacia; afrontamiento 


\section{ABSTRACT}

Objectives: To assess emotional variables (anxiety, depression), cognitive variables (catastrophizing, perceived self-efficacy) and behavioral variables (coping); to know in what way these kind of variables influence health-related quality of life in people with chronic conditions; analyze if there are significant differences depending on gender or chronic disease diagnosis on psychological variables mentioned; to detect if significant differences exist in the different dimensions of health-related quality of life between normal population and people with chronic disease.

Method: Descriptive-correlational study made in February and March 2015 in which 35 people participated with different diagnoses of chronic disease. 24 of them $(68.6 \%)$ were female and 11 (31.4\%) were males.

Results: The results obtained suggest that emotional variables affect health-related quality of life, as well as perceived self-efficacy and coping strategies. We also find that people who was assessed showed significantly lower levels in health-related quality of life than general population, and that it seem to exist few differences in the variables studied between people with different diagnosis and between men and women.

Conclusions: Psychological variables play an important role in the quality of life of people with chronic diseases. This shows the importance of addressed these kind of aspects. The results are consistent with others found in previous studies. Further research is needed with larger samples.

Keywords: chronic disease; health-related quality of life; self-efficacy; coping

\section{INTRODUCCIÓN}

A lo largo de las últimas décadas, hemos pasado de una mayor prevalencia de enfermedades infecciosas a la situación actual, en la que las enfermedades crónicas (EC) son las responsables del $59 \%$ de las muertes y del $46 \%$ de la carga de enfermedad en todo el mundo ${ }^{(1)}$. Cuando hablamos de condiciones físicas crónicas, nos referimos a un término que abarca las llamadas enfermedades no transmisibles (como el asma, artritis, diabetes, cardiopatía isquémica, etc.) y algunas enfermedades transmisibles (como el VIH/SIDA) ${ }^{(2)}$.

A diferencia de las patologías de carácter agudo, este tipo de condiciones persisten a lo largo de la vida, teniendo múltiples consecuencias en las distintas áreas de la vida de la persona, provocando deterioro en el funcionamiento de quienes las padecen, lo cual puede repercutir en su autocuidado, en las actividades de la vida diaria, en su tiempo de ocio. También pueden suponer consecuencias a nivel laboral, aumentando la cantidad de bajas en el trabajo e incluso de jubilaciones por incapacidad. Por ejemplo, en el caso de la diabetes en nuestro país, en 2011 se produjeron 2567 procesos de incapacidad temporal debidos a la enfermedad ${ }^{(3)}$.

Asimismo, las condiciones físicas crónicas tienen repercusiones fundamentales a nivel emocional, económico, familiar y social. Tomando como punto de referencia las puntuaciones obtenidas en el cuestionario SF-36 se puede ver como se da una importante afectación negativa en la calidad de vida de personas con diagnósticos diversos como espondilitis anquilosante, artritis psoriásica, artritis reumatoide, lupus eritematoso sistémico, esclerosis múltiple, síndrome de Sjögren y fibromialgia ${ }^{(4,5)}$.

Lo expuesto previamente, evidencia la necesidad de utilizar el modelo biopsicosocial de comprensión del funcionamiento, la discapacidad y la calidad de vida en personas con EC. El modelo considera que los aspectos físicos propios de la enfermedad no se producen aislados sino que interactúan de forma compleja con factores psicológicos 
(emocionales, cognitivos y conductuales) y sociales. $Y$ es que los factores físicos no resultan suficientes para una comprensión adecuada de la calidad de vida de los pacientes. Por ejemplo, la revisión de García Sanjuan et al. ${ }^{(6)}$ pone de manifiesto la necesidad de realizar investigaciones de carácter más integrador en relación a los cuidados a personas con EC. De hecho, a lo largo de los últimos años, han ido surgiendo cada vez más estudios que resaltan el papel de las variables psicosociales en las EC. No obstante, hasta donde llega nuestro conocimiento, hay pocos estudios que aborden en profundidad las distintas variables psicológicas comunes asociadas a varias condiciones crónicas. Por el contrario, las investigaciones tienden a ser específicas de enfermedades concretas (enfermedad pulmonar, enfermedad renal, etc.). Sin embargo, la revisión de la literatura realizada por Swendeman et al. ${ }^{(7)}$ pone de manifiesto la existencia de elementos comunes de cara al manejo de la enfermedad.

En relación con aspectos emocionales, la evidencia empírica muestra que la asociación entre EC y estados emocionales negativos es clara. Por ejemplo, en un estudio de Teesson et al. ${ }^{(8)}$ en población australiana, se encontró que en personas con enfermedades físicas crónicas, alrededor de un $46 \%$ padecía problemas de ansiedad y un $42.6 \%$ de depresión. Diferentes estudios sugieren que la influencia de estos factores es comparable al de las propias EC en sí, contribuyendo a aumentar los efectos negativos de estas en la calidad de vida relacionada con la salud ${ }^{(9,10)}$. En este sentido, Egede ${ }^{(11)}$ estima que los pacientes que padecen depresión y enfermedades físicas crónicas tienen 2.5 veces más probabilidades de presentar discapacidad que aquellos sin sintomatología depresiva.

Sin embargo, no son los síntomas emocionales los únicos asociados a las EC. Los pensamientos y creencias negativas, como el catastrofismo o la baja autoeficacia percibida, son comunes en este tipo de condiciones. Las creencias negativas como el catastrofismo están fuertemente asociadas con mayores limitaciones físicas, niveles más elevados de dolor y más necesidad de analgésicos que en iguales con menor nivel de catastrofismo ${ }^{(12)}$. En cuanto a la autoeficacia, que consiste en la percepción que tiene la persona de su capacidad para hacer frente a la situación, desempeña un papel fundamental, ya que si una persona se percibe incapaz de hacer nada en relación a la enfermedad ello interfiere en el afrontamiento de la misma.

Por su parte, los aspectos conductuales de la enfermedad también son relevantes. Algunos de estos factores que influyen en los resultados de funcionamiento y calidad de vida incluyen las conductas llevadas a cabo por las personas y las estrategias de afrontamiento utilizadas para abordar los síntomas y consecuencias de la enfermedad. Así, por ejemplo, la inactividad física es común en los pacientes crónicos, que debido a sus síntomas tienden a reducir su actividad a veces incluso a niveles mínimos. Las repercusiones de este tipo de conducta tienen efectos contraproducentes, ya que la inactividad empeora el estado de ánimo, la fatiga, la calidad de vida y la salud en general. Se ha comprobado que el modo en que los pacientes afrontan su enfermedad influye más en su calidad de vida que el tipo de EC que tienen ${ }^{(13)}$. Un afrontamiento activo, basado en la resolución de problemas, está asociado con menores niveles de dolor, menor deterioro en el funcionamiento y autoeficacia percibida más alta que un estilo de afrontamiento más pasivo ${ }^{(14)}$.

Como puede verse, los síntomas emocionales, cognitivos y conductuales están asociados a las EC hasta el punto de que pueden suponer algunas de las complicaciones más comunes en este tipo de condiciones, repercutiendo 
negativamente en la calidad de vida, las habilidades de automanejo de la enfermedad y la comunicación con los profesionales de la salud. A esto hay que añadir que las repercusiones psicosociales de la enfermedad intensifican el dolor, la fatiga y el aislamiento social, entre otros ${ }^{(15)}$.

Partiendo del marco conceptual previo y siendo conscientes de la importancia de distintas variables psicológicas en estas poblaciones nos planteamos realizar un estudio para describir y analizar dichas variables en personas con EC de la provincia de Salamanca. En concreto, nos planteamos los siguientes objetivos:

- Describir la calidad de vida relacionada con la salud, aspectos emocionales (ansiedad, depresión), estrategias de afrontamiento y niveles de autoeficacia percibida en personas con EC de Salamanca.

- Describir las relaciones entre las distintas variables evaluadas.

- Identificar cuáles son las variables que más contribuyen a explicar la varianza de la calidad de vida percibida.

Las hipótesis planteadas son las siguientes: 1) Los participantes en el estudio percibirán tener bajos niveles de calidad de vida, significativamente inferiores a la población general; 2) Se obtendrán niveles elevados de depresión y ansiedad; 3) No se obtendrán diferencias significativas en función del tipo de enfermedad en las variables evaluadas; 4) No se encontrarán diferencias significativas entre varones y mujeres en las distintas variables evaluadas; 5) Se obtendrán asociaciones negativas entre la calidad de vida percibida y variables emocionales como la ansiedad y depresión.

\section{MÉTODO}

A fin de realizar una descripción de las variables implicadas en los objetivos, así como de analizar las relaciones existentes entre las mismas se llevó a cabo un estudio descriptivo-correlacional de corte transversal.

\section{Participantes}

En el presente estudio participaron, en los meses de febrero y marzo de 2015, un total de 35 personas pertenecientes a distintas asociaciones de EC de Salamanca, de las cuales $24(68.6 \%)$ eran mujeres y 11 (31.4\%) varones. Las edades estaban comprendidas entre 31 y 88 años $(M=54.91$; $D T=13.42)$. Presentaban distintos diagnósticos, como lupus, artritis reumatoide, enfermedad pulmonar, fibromialgia, espondilitis anquilosante, colitis ulcerosa, enfermedad de Crohn, insuficiencia renal y esclerosis múltiple, entre otras. La media de tiempo desde que se les diagnosticó la enfermedad fue de 13.3 años (DT=11.41).

\section{Variables, instrumentos de evaluación y materiales}

Se evaluaron las siguientes variables de interés: calidad de vida relacionada con la salud, depresión, ansiedad (estado y rasgo), estrategias de afrontamiento ante situaciones estresantes y ante situaciones específicas de dolor y autoeficacia percibida para controlar la enfermedad y sus repercusiones. Se utilizaron los siguientes instrumentos de evaluación ${ }^{(16-22)}$ : inventario de depresión de Beck (BDI), 
cuestionario de ansiedad estado-rasgo (STAI), cuestionario SF-36 de calidad de vida relacionada con la salud, escala de autoeficacia percibida para enfermedad crónica (CDSES), Inventario de Estrategias de Afrontamiento_(CSI), cuestionario de afrontamiento del dolor crónico (CAD), y cuestionario de estrategias de afrontamiento del dolor (CSQ).

\section{Procedimiento}

En primer lugar se contactó con los responsables del área de promoción de la Salud del Ayuntamiento de Salamanca, informándoles del proyecto dirigido a las Asociaciones de personas con enfermedades crónicas. Una vez fue posible, se procedió a citar en los locales de la Casa de las Asociaciones de ayuda mutua a las personas interesadas en colaborar. Un total de 35 personas cumplimentaron, previo consentimiento informado, los distintos instrumentos de evaluación, además de un cuestionario sociodemográfico.

\section{Análisis estadísticos}

Los análisis se realizaron con el programa de análisis de datos SPSS (versión 21) y Microsoft Excel (2007). En primer lugar se realizaron análisis exploratorios de las variables. En relación con los objetivos propuestos, se realizó en primer lugar un análisis descriptivo de las variables evaluadas. Para comprobar la existencia de diferencias significativas en las variables evaluadas en función del género se utilizó la prueba $t$ de diferencia de medias para muestras independientes, y para valorar la existencia de diferencias significativas en función del tipo de enfermedad crónica se utilizó la prueba no paramétrica Kruskal-Wallis (pues algunos grupos constaban de un número muy reducido de sujetos). Para comprobar si existían diferencias significativas en calidad de vida percibida entre los participantes del estudio y la muestra de población general española del estudio realizado por Alonso et al. ${ }^{(23)}$ se utilizó la prueba $t$ de Student para muestras independientes, calculando después la $d$ de Cohen para estimar el tamaño del efecto. Por otro lado, con el objetivo de comprobar si existía asociación entre las variables se realizaron correlaciones bivariadas entre las distintas variables, utilizando el coeficiente de correlación de Pearson. Finalmente, a fin de determinar el poder predictivo de las variables consideradas conjuntamente sobre la calidad de vida, se realizaron diversos análisis de regresión múltiple (método stepwise) tomando en cada uno de ellos como variable dependiente una dimensión de calidad de vida del SF-36. Los predictores se introdujeron por bloques, así en el primer bloque se incluyeron variables emocionales como depresión y ansiedad (estado y rasgo); en el segundo bloque las estrategias de afrontamiento evaluadas por el CSQ, en el tercero las estrategias de afrontamiento evaluadas por el CAD, finalmente en el último bloque se introdujo la autoeficacia percibida.

\section{RESULTADOS}

A nivel descriptivo (ver tabla I), las puntuaciones medias obtenidas por el grupo indican la existencia de niveles leves de depresión y ansiedad, así como una moderada autoeficacia percibida. En cuanto a la calidad de vida relacionada con la salud, las medias obtenidas en el SF-36 tienden a ser bajas en todas las dimensiones, destacando como las más bajas rol físico (es decir, la medida en que los problemas físicos impiden llevar a cabo las actividades cotidianas o deseadas), salud general, dolor corporal, funcionamiento social y vitalidad. Las estrategias de afrontamiento más utilizadas son ignorar el dolor, uso de conductas distractoras, autoinstrucciones y 
acudir a la religión, mientras que las menos empleadas son autocrítica, evitación de problemas y esperanza. Otras estrategias de afrontamiento como distracción cognitiva, reinterpretar el dolor, reestructuración cognitiva, autocontrol mental y resolución de problemas, son utilizadas de forma moderada.

Tabla I. Descriptivos de las variables (Tabla de M y DT)

\begin{tabular}{|c|c|c|}
\hline DIMENSIONES SF-36 & $\mathrm{N}$ & $\mathrm{M}(\mathrm{DT})$ \\
\hline FUNCIONAMIENTO FISICO $(0-100)$ & 35 & $42.92(26.25)$ \\
\hline ROL FISICO $(0-100)$ & 34 & $27.94(42.98)$ \\
\hline DOLOR CORPORAL $(0-100)$ & 35 & $42.89(28.12)$ \\
\hline SALUD GENERAL $(0-100)$ & 35 & $32.74(22.12)$ \\
\hline VITALIDAD $(0-100)$ & 35 & $45.52(23.23)$ \\
\hline FUNCIONAMIENTO SOCIAL $(0-100)$ & 35 & $57.14(24.12)$ \\
\hline ROL EMOCIONAL $(0-100)$ & 35 & $63.81(42.30)$ \\
\hline SALUD MENTAL $(0-100)$ & 35 & $63.51(19.46)$ \\
\hline COMPONENTE FISICO ESTANDARIZADO $(0-100)$ & 34 & $30.35(11.97)$ \\
\hline COMPONENTE MENTAL ESTANDARIZADO $(0-100)$ & 34 & $45.63(11.96)$ \\
\hline VARIABLES EMOCIONALES Y AUTOEFICACIA & $\mathrm{N}$ & $\mathrm{M}(\mathrm{DT})$ \\
\hline$\overline{\text { DEPRESION }}$ & 32 & $13.34(7.97)$ \\
\hline ANSIEDAD ESTADO & 30 & $23.10(11.01)$ \\
\hline ANSIEDADRASGO & 29 & $29.97(8.17)$ \\
\hline AUTOERICACIA & 33 & $20.61(7.84)$ \\
\hline ESTRATEGIAS DE AFRONTAMIENTO & $\mathrm{N}$ & $\mathrm{M}(\mathrm{DT})$ \\
\hline CA TASTROFISMO & 31 & $13.35(6.94)$ \\
\hline CONDUCTAS DISTRA CTORAS CSQ & 32 & $19.53(7.42)$ \\
\hline AUTOINSTRUCCIONES & 27 & $17.19(5.88)$ \\
\hline IGNORAR EL DOLOR & 31 & $20.58(7.43)$ \\
\hline REINTERPRETAR EL DOLOR & 31 & $14.87(6.25)$ \\
\hline ESPERANZA & 32 & $9.69(4.64)$ \\
\hline FB Y PLEGARIAS & 32 & $6.44(6.00)$ \\
\hline DISTRACCION COGNITIVA & 31 & $11.48(3.71)$ \\
\hline RELIGION & 31 & $16.94(7.65)$ \\
\hline $\begin{array}{l}\text { CA TARSIS (BUSQUEDA DE APOYO SOCIAL } \\
\text { EMOCIONAL) }\end{array}$ & 31 & $15.97(4.74)$ \\
\hline DISTRACCION CAD & 32 & $15.34(5.03)$ \\
\hline AUTOCONTROL MENTAL & 33 & $15.33(4.10)$ \\
\hline AUTOAFIRMACION & 33 & $10.55(4.18)$ \\
\hline $\begin{array}{l}\text { BU SQUEDA DE INFORMACION (APOYO SOCIAL } \\
\text { INSTRUMENTAL) }\end{array}$ & 33 & $11.67(3.08)$ \\
\hline RESOLUCION DE PROBLEMAS & 32 & $13.13(4.37)$ \\
\hline AUTOCRITICA & 33 & $6.15(5.23)$ \\
\hline EXPRESIÓN EMOCIONAL & 34 & $9.15(3.92)$ \\
\hline PENSAMIENTO DESIDERATTVO & 32 & $12.94(5.07)$ \\
\hline APOYO SOCIAL & 29 & $10.62(4.72)$ \\
\hline REE STRUCTURACION COGNITIVA & 32 & $10.63(4.76)$ \\
\hline BVITACION DE PROBLEMAS & 35 & $8.06(3.28)$ \\
\hline RETIRADA SOCIAL & 34 & $8.91(3.87)$ \\
\hline
\end{tabular}

Los resultados de la prueba $t$ indican la existencia de diferencias significativas entre hombres y mujeres, a favor de los primeros en algunas dimensiones de calidad de vida como dolor corporal $[t=-2.267, p=.030 ; M(D T)$ (hombres) $=57.9$ (31.18); $M(D T)$ (mujeres) $=36(24.26)], \quad$ funcionamiento social $[t=-2.594, \quad p=.014 ; \quad M(D T)$ (hombres)=71.59 (18.62); $\mathrm{M}(\mathrm{DT})$ (mujeres)=50.52(23.74)] y el componente mental estandarizado $\quad[t=-2.309, \quad p=.028 ; \quad M(D T) \quad$ (hombres) $=52.06 \quad(42.55) ; \quad M(D T)$ (mujeres) $=8.21(12.38)]$. No se encontraron diferencias en el resto de variables.

Respecto al tipo de enfermedad, los resultados de la prueba Kruskal-Wallis mostraron diferencias significativas entre los distintos grupos en las variables catastrofismo $\left[X^{2}(6)=15.14, p=.049\right]$ y rol emocional del SF-36 $\left[X^{2}(6)=15.83, p=.027\right]$. En la figura 1 se pueden ver gráficamente las medias de los distintos grupos en estas variables. En cuanto al resto de variables, no se encontraron diferencias significativas. 
Figura 1. Puntuaciones medias en rol emocional y catastrofismo según diagnóstico de enfermedad crónica

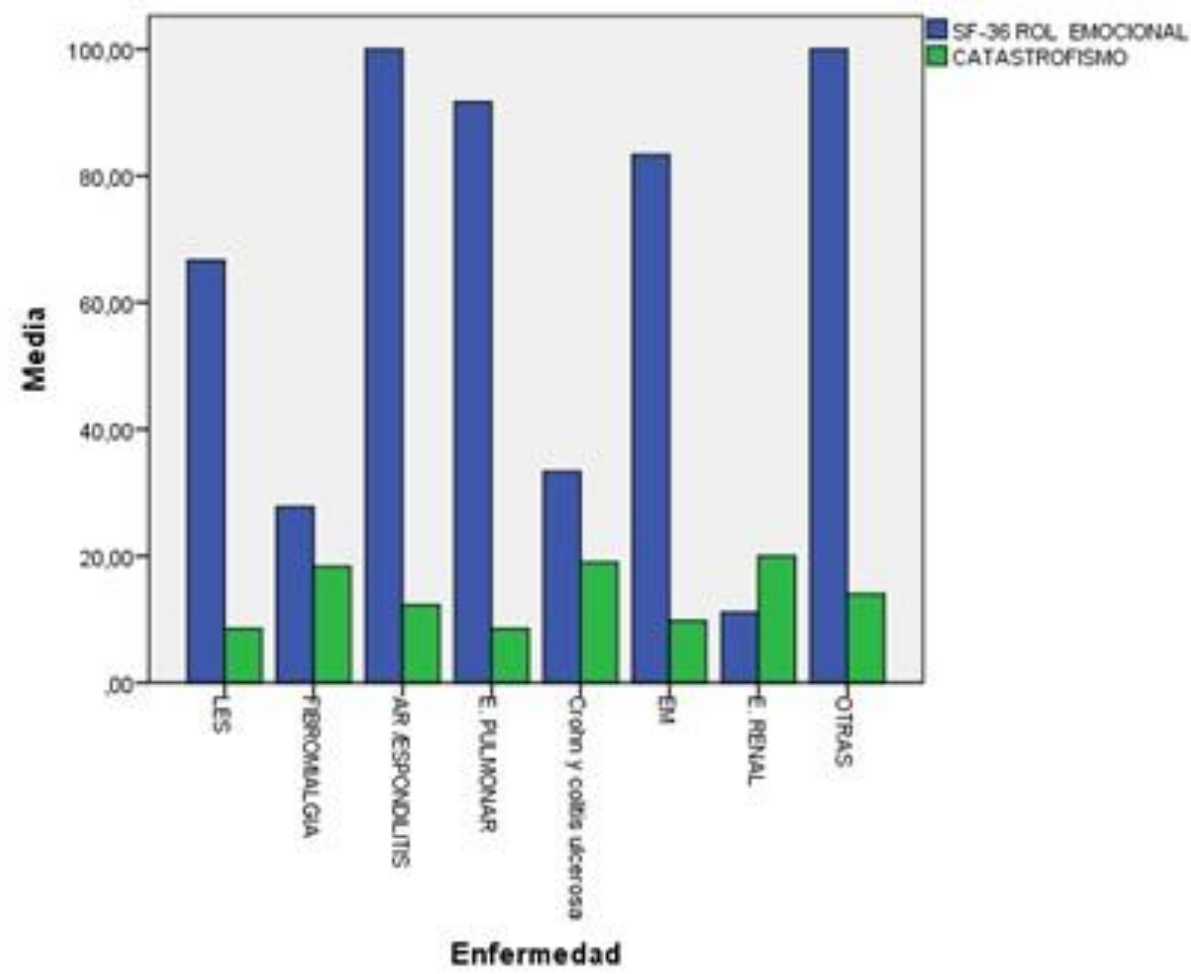

Los resultados de la prueba t de comparación entre el grupo de participantes y la población general indican la existencia de diferencias significativas en todas las variables (véase la tabla II). Como se puede observar en la figura 2, son los participantes de nuestro estudio quienes obtienen puntuaciones significativamente más bajas en todas las dimensiones de calidad de vida, con un tamaño del efecto $\mathrm{d}>0.8$ (efecto grande) en las variables funcionamiento físico, rol físico, dolor corporal, salud general, vitalidad y funcionamiento social, y un tamaño del efecto medio en las variables salud mental $(\mathrm{d}=0.51)$ y rol emocional $(\mathrm{d}=0.69)$.

Tabla 2. Medias y desviaciones típicas en las dimensiones del SF-36 en personas con enfermedad crónica y población normal del estudio de Alonso y cols. (1998)

Población normal Pacientes con enfermedad crónica

\begin{tabular}{llll}
\hline & M (DT) & M(DT) & \multicolumn{1}{c}{ T } \\
\hline Funcionamiento físico & $84.70(24.00)$ & $42.92(26.25)$ & $-9.26476735^{*}$ \\
Rol físico & $83.20(35.20)$ & $27.94(42.98)$ & $-7.4863946^{*}$ \\
Dolor corporal & $79.00(22.30)$ & $42.89(28.12)$ & $-7.47780409^{*}$ \\
Salud general & $68.30(22.30)$ & $32.74(22.12)$ & $-9.35463828^{*}$ \\
Vitalidad & $66.90(22.10)$ & $45.52(23.23)$ & $-5.35671179^{*}$ \\
Funcionamiento social & $90.10(20.00)$ & $57.14(24.12)$ & $-7.95654672^{*}$ \\
Rol emocional & $88.60(30.10)$ & $63.81(42.3)$ & $-3.41347479^{*}$ \\
Salud mental & $73.30(20.10)$ & $63.51(19.46)$ & $-2.92718141^{*}$ \\
\hline$* \mathrm{p}<.05$ & & & \\
\hline
\end{tabular}


Figura 2. Puntuaciones medias en las dimensiones del SF-36 de personas con enfermedad crónica y población normal del estudio de Alonso y cols. (1998)

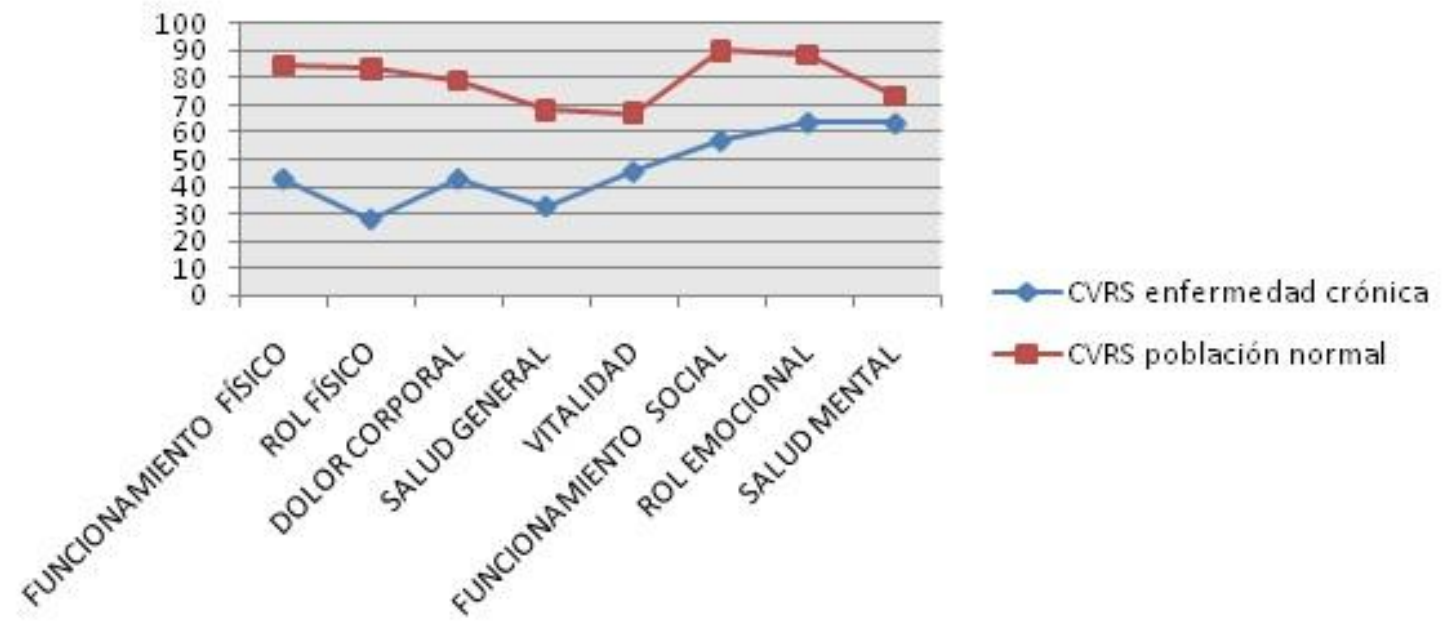

Los resultados del análisis de regresión lineal múltiple mostraron que algunas de las variables evaluadas tenían cierto poder predictivo sobre la calidad de vida relacionada con la salud. Según los resultados obtenidos, las variables depresión, ansiedad (estado y rasgo), autoeficacia, distracción cognitiva y conductas distractoras predicen el resultado de algunas dimensiones de calidad de vida, estando las variables emocionales negativamente asociadas a la misma, mientras que la autoeficacia y las estrategias de distracción cognitiva y conductas distractoras estarían positivamente asociadas a ella. Aunque en las tablas III, IV y V pueden verse los resultados del análisis de forma detallada, cabe destacar algunos resultados. Por ejemplo, la ansiedad rasgo explica por sí sola el $40.7 \%$ de la varianza de funcionamiento social, y el $55.7 \%$ junto con la depresión. Por otro lado, la ansiedad estado junto con la autoeficacia percibida y el uso de conductas distractoras explicarían el $64,8 \%$ de la varianza de la dimensión salud mental. En cuanto al componente físico de calidad de vida, la depresión explica el $48.8 \%$ de la varianza. 
Tabla III. Análisis de regresión por bloques (salud mental, funcionamiento social y vitalidad)

\begin{tabular}{|c|c|c|c|c|c|c|c|c|c|c|c|}
\hline \multicolumn{4}{|c|}{ SALUD MENTAI } & \multicolumn{4}{|c|}{ FUNCIONAMIENTO SOCIAL } & \multicolumn{4}{|c|}{ VITALIDAD } \\
\hline $\begin{array}{l}\text { Mo } \\
\text { d. }\end{array}$ & $\mathrm{R}$ & $\mathrm{R}^{2}$ & $\mathrm{R}^{2}$ ajusta do & Mod. & $\mathrm{R}$ & $\mathrm{R}^{2}$ & $\begin{array}{l}\mathrm{R}^{2} \\
\text { ajustado }\end{array}$ & Mod. & $\mathrm{R}$ & $\mathrm{R}^{2}$ & $\begin{array}{l}\mathrm{R}^{2} \\
\text { ajusta } \\
\text { do }\end{array}$ \\
\hline 1 & 491 & 241 &, 196 & 1 &, 638 & 407 & 372 & 1 &, 701 & 491 & 461 \\
\hline 2 &, 697 & 486 & 421 & 2 &, 746 & 557 &, 501 & 2 & .788 &, 621 &, 573 \\
\hline 3 &, 805 &, 648 &, 577 & & & & & & & & \\
\hline $\begin{array}{l}\text { Mo } \\
\text { d. }\end{array}$ & & & Beta & Mod. & & & Beta & Mod. & & & Beta \\
\hline 1 & \multicolumn{2}{|c|}{$\begin{array}{l}\text { Arsiedad } \\
\text { Estado }\end{array}$} &,$- 491^{*}$ & 1 & \multicolumn{2}{|c|}{$\begin{array}{l}\text { Ansiedad } \\
\text { Rasgo }\end{array}$} &,$- 638 * 4$ & 1 & \multicolumn{2}{|c|}{$\begin{array}{l}\text { Ansiedad } \\
\text { Rasgo }\end{array}$} &,$- 701^{44}$ \\
\hline \multirow[t]{3}{*}{$\overline{2}$} & \multicolumn{2}{|c|}{ (Constante) } & & 2 & \multicolumn{2}{|c|}{ (Constante) } & & 2 & \multicolumn{2}{|c|}{ (Constante) } & \\
\hline & \multicolumn{2}{|c|}{ Ansiedad } & $\begin{array}{l}-, 664^{4}+ \\
524^{*}\end{array}$ & & \multicolumn{2}{|c|}{$\begin{array}{l}\text { Ansiedad } \\
\text { Rasgo }\end{array}$} &,$- 489^{4}$ & & \multicolumn{2}{|c|}{$\begin{array}{l}\text { Ansiedad } \\
\text { Rasgo }\end{array}$} &,$- 484^{4}$ \\
\hline & \multicolumn{2}{|c|}{$\begin{array}{l}\text { Distracción } \\
\text { cognitiva }\end{array}$} & & & \multicolumn{2}{|c|}{ Depresión } &,$- 414^{*}$ & & \multicolumn{2}{|c|}{ Avtoeficacia } & $420^{4}$ \\
\hline \multirow{3}{*}{3} & \multicolumn{2}{|c|}{$\begin{array}{l}\text { Arsiedad } \\
\text { Estado }\end{array}$} & -184 & & & & & & & & \\
\hline & \multicolumn{2}{|c|}{$\begin{array}{l}\text { Distracción } \\
\text { cognitiva }\end{array}$} & 219 & & & & & & & & \\
\hline & \multicolumn{2}{|c|}{ Autoeficacia } & $624^{4}$ & & & & & & & & \\
\hline
\end{tabular}

Tabla IV. Análisis de regresión por bloques (salud general, rol físico y funcionamiento físico)

\begin{tabular}{|c|c|c|c|c|c|c|c|c|c|c|c|}
\hline \multicolumn{4}{|c|}{ SALUD GENERAL } & \multicolumn{4}{|c|}{ ROL FÍSICO } & \multicolumn{4}{|c|}{ FUNCIONAMIENTO FÍSICO } \\
\hline $\begin{array}{l}\text { Mo } \\
\text { d. }\end{array}$ & $\mathrm{R}$ & $\mathrm{R}^{2}$ & $\mathrm{R}^{2}$ ajustado & Mod. & $\mathrm{R}$ & $\mathrm{R}^{2}$ & $\begin{array}{l}\mathrm{R}^{2} \\
\text { ajusta } \\
\text { do }\end{array}$ & Mod. & $\mathrm{R}$ & $\mathrm{R}^{2}$ & $\begin{array}{l}\mathrm{R}^{2} \\
\text { ajusta } \\
\text { do }\end{array}$ \\
\hline \multirow[t]{2}{*}{1} & \multirow[t]{2}{*}{,631 } & \multirow[t]{2}{*}{,398 } & \multirow[t]{2}{*}{,362 } & 1 & ,673 & 452 & ,420 & \multirow[t]{2}{*}{1} & \multirow[t]{2}{*}{470} & \multirow[t]{2}{*}{,221 } & \multirow[t]{2}{*}{, 175} \\
\hline & & & & 2 & ,787 & 620 & ,573 & & & & \\
\hline $\begin{array}{l}\text { Mo } \\
\text { d. }\end{array}$ & & & Beta & Mod. & & & Beta & Mod. & & & Beta \\
\hline \multirow[t]{3}{*}{1} & \multirow{3}{*}{\multicolumn{2}{|c|}{$\begin{array}{l}\text { (Constante) } \\
\text { Ansiedad } \\
\text { Rasgo }\end{array}$}} & \multirow{3}{*}{,$- 631 * *$} & 1 & (Cons & & & 1 & \multirow{3}{*}{\multicolumn{2}{|c|}{$\begin{array}{l}\text { (Constante) } \\
\text { Ansiedad } \\
\text { Estado }\end{array}$}} & \\
\hline & & & & & $\begin{array}{l}\text { Ansie } \\
\text { Estad }\end{array}$ & &,$- 673 *$ & & & &,$- 470^{*}$ \\
\hline & & & & 2 & $\begin{array}{l}\text { (Cons } \\
\text { Ansie } \\
\text { Estad } \\
\text { Cond } \\
\text { distra }\end{array}$ & & $\begin{array}{l}-, 744 * \\
, 416 * *\end{array}$ & & & & \\
\hline
\end{tabular}


Tabla V. Análisis de regresión por bloques (componente físico y mental de calidad de vida)

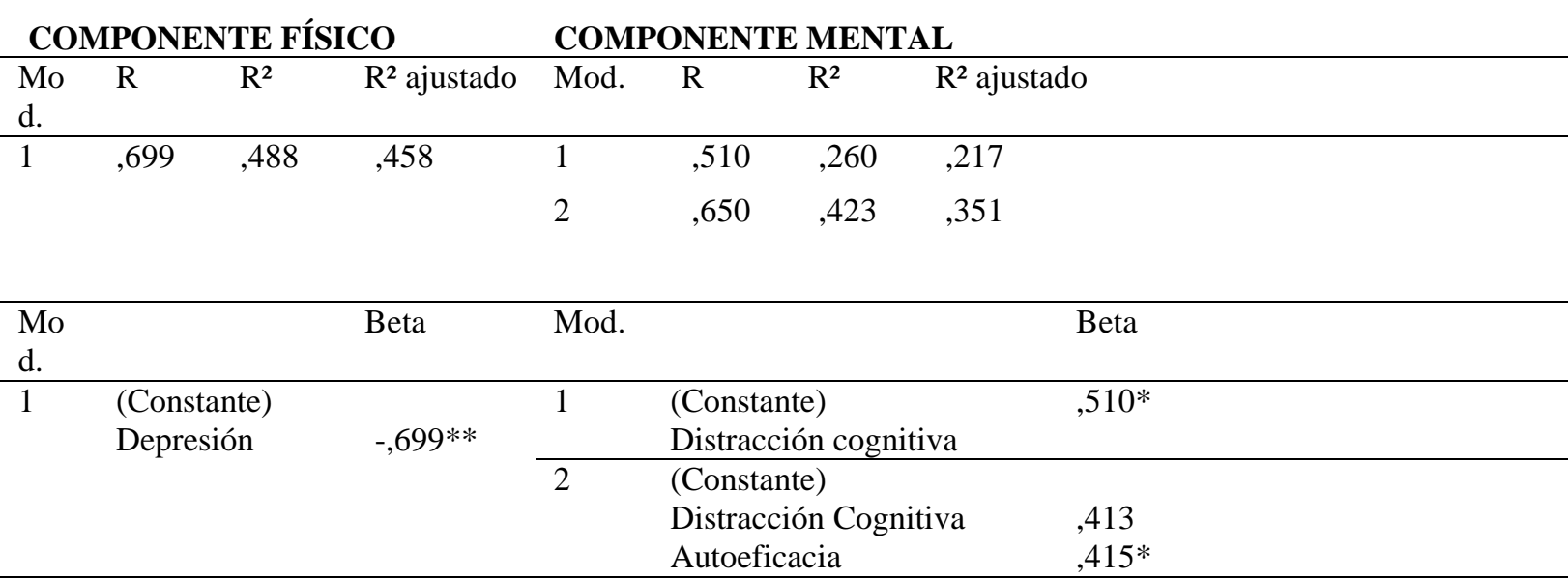

$* \mathrm{p}<.05 * * \mathrm{p}<.01$

\section{DISCUSIÓN}

En primer lugar, tal como sugieren los resultados obtenidos en el SF-36, la calidad de vida relacionada con la salud de las personas con enfermedad crónica es baja, lo cual es acorde a lo que se ha puesto de manifiesto a lo largo de la literatura científica. Sin embargo, no todos los estudios coinciden en qué dimensiones se ven más afectadas. Mientras que en nuestro caso las puntuaciones más bajas se dan en funcionamiento físico, rol físico y salud general, los resultados de otros estudios como es el caso de González et al. ${ }^{(9)}$ muestran la vitalidad como una de las más bajas. En cuanto a salud general, los estudios suelen coincidir en que es una de las dimensiones más afectadas, como ponen de manifiesto los resultados de Hincapié et al. ${ }^{(5)}$ en personas con esclerosis múltiple, lupus eritematoso sistémico, artritis reumatoide y espondilitis anquilosante, entre otros.

Respecto a la comparación de los resultados del SF-36 del estudio de Alonso et al. ${ }^{(23)}$, las diferencias existentes entre la población normal y la muestra de este estudio de personas con enfermedad crónica son evidentes. Este resultado es consistente con el estudio de Hervás et al. ${ }^{(24)}$ sobre personas con diabetes tipo 2, en el que destacan las diferencias con la población general en las dimensiones funcionamiento físico y salud general, pero difiere de nuestros resultados en aspectos como rol físico, vitalidad y salud mental, en los que no muestran diferencias respecto a la población general.

Asimismo, los resultados sugieren la importancia de las variables emocionales, cognitivas y conductuales evaluadas. En primer lugar, las puntuaciones obtenidas sugieren la existencia de niveles leves de depresión, algo que es consistente con otros estudios que muestran cómo diversos tipos de pacientes con enfermedades crónicas obtienen puntuaciones en el BDI que indican sintomatología depresiva levemoderada como es el caso de personas con enfermedad renal ${ }^{(25)}$ o el de personas con enfermedad pulmonar ${ }^{(26)}$. También los resultados obtenidos en ansiedad son similares a otros estudios en los que se encuentra ansiedad moderada 0 moderadamente alta, como es el caso de los pacientes con enfermedad renal crónica del estudio de Theofilou ${ }^{(27)}$. Sin embargo, este último, a pesar de que al igual que nuestro estudio no encuentra diferencias significativas en función del género en 
depresión y en ansiedad rasgo sí lo hace en ansiedad estado, presentando puntuaciones mayores las mujeres.

Asimismo, las estrategias de afrontamiento utilizadas con más frecuencia, como las conductas distractoras o las autoinstrucciones, pueden suponer un esfuerzo por parte de los pacientes por tratar de llevar una vida activa y seguir tomando el control a pesar de la enfermedad. No obstante, otras estrategias que tienden a resultar adaptativas, como la distracción cognitiva, reinterpretar el dolor, la reestructuración cognitiva, el autocontrol mental y la resolución de problemas, son utilizadas de forma moderada, por lo que convendría incrementar su puesta en práctica. Por otra parte, aunque algunos autores como Soucase et al. ${ }^{(28)}$ manifiestan que la autoafirmación y la búsqueda de apoyo social instrumental son estrategias de afrontamiento de uso frecuente, en nuestro estudio no son las más utilizadas. Asimismo, en otras investigaciones como la de Almeida \& $\operatorname{Rivas}^{(29)}$ realizada con pacientes con diabetes tipo 2, la autocrítica, la evitación de problemas, la retirada social y el pensamiento desiderativo son las estrategias más comunes, mientras que en nuestro estudio si bien las tres primeras son de las menos utilizadas, el pensamiento desiderativo aparece con frecuencia como estrategia utilizada.

Cabe destacar que, salvo contadas excepciones, los resultados reflejan la ausencia de diferencias significativas entre las variables en función de las distintas enfermedades estudiadas. Esto está en consonancia con la revisión de la literatura de Swendeman et al. ${ }^{(7)}$ que pone de manifiesto que hay elementos comunes en el automanejo de la enfermedad entre los distintos tipos de condiciones y justifica el hecho de que se puedan abordar en conjunto en programas de autogestión como el propuesto en este trabajo.

Asimismo, tal y como sugiere el análisis de regresión en este estudio las variables emocionales, la autoeficacia y estrategias de afrontamiento como conductas distractoras o distracción cognitiva muestran un poder predictivo de la calidad de vida percibida que merece ser tomado en consideración. Estos resultados coinciden con los de Vilhena et al. ${ }^{\left({ }^{(0)}\right.}$ en cuanto a la importancia del bienestar emocional en la calidad de vida percibida.

Como conclusión se puede decir que los resultados obtenidos muestran la importancia que toman en las personas con EC no solo los síntomas físicos, sino también los síntomas emocionales, la autoeficacia y las estrategias de afrontamiento, al tiempo que ponen de manifiesto el bajo nivel de calidad de vida relacionada con la salud en este tipo de pacientes.

Asimismo, el hecho de que no se hayan encontrado diferencias entre las distintas variables evaluadas entre las distintas enfermedades tiene implicaciones importantes, pues contribuye al hecho de que al realizar programas de automanejo de la enfermedad los grupos puedan estar compuestos de personas con distintos diagnósticos de enfermedad crónica.

Dadas las limitaciones del estudio, como el reducido tamaño de la muestra, estos resultados han de ser tomados con cautela. En futuras investigaciones deberán utilizarse tamaños muestrales más amplios. Además, dado que la recogida de datos en ambos estudios ha sido realizada únicamente con miembros de asociaciones de enfermedades crónicas, sería recomendable que futuros trabajos se realizasen con 
más muestras con este tipo de patologías (como por ejemplo, en contextos hospitalarios o de atención primaria).

En definitiva, y basándonos en lo expuesto previamente, las implicaciones de este estudio merecen ser tomadas en consideración dada la importancia de este tipo de enfermedades y del impacto de los aspectos estudiados a nivel no solo personal, sino también familiar, económico y social.

\section{Agradecimientos}

A todos los pacientes de las asociaciones de enfermedades crónicas que, desinteresadamente, accedieron a participar en el estudio. También agradecer las facilidades y los recursos aportados a los responsables de la Casa de las Asociaciones del Ayuntamiento de Salamanca.

\section{REFERENCIAS}

1. Fortin $M$, Chouinard $M C$, Bouhali $T$, Dubois $M$, Gagnon $C$, \& Bélanger $M$. Evaluating the integration of chronic disease prevention and management services into primary health care. BMC Health Services Research 2013; 13(1), 132.

2. Organização Mundial da Saúde (OMS). Cuidado innovador para las condiciones crónicas: agenda para el cambio. Ginebra: OMS. 2002.

3. Vicente-Herrero MT, Terradillos MJ, Capdevila LM, Ramírez Iñiguez M, \& LópezGonzález ÁA. Costs of temporary disability in Spain related to diabetes mellitus and its complications. Endocrinología Y Nutrición (English Edition) 2013; 60(8), 447-455.

4. Freire M, Rodríguez J, Möller I, Valcárcel A, Tornero C, Díaz G, Armendazáriz Y, \& Paredes S. Prevalencia de síntomas de ansiedad y de depresión en pacientes con artritis psoriásica en consultas de reumatología. Reumatología Clínica 2011; 7(1), 20-26. doi:10.1016/j.reuma.2010.03.003

5. Hincapié ME, Suárez JC, Pineda R, \& Anaya JM. Calidad de vida en esclerosis múltiple y otras enfermedades crónicas autoinmunes y no autoinmunes. Revista de Neurología 2009; 48(5). Disponible en: http://www.coloquiodiscapacidad.com/investigaciones/phocadownload/Salud-viday-funcionamiento-humano/hincapie_otros_2009.pdf

6. García Sanjuan S, Lillo Crespo, $\bar{M}$, Sanjuan Quiles, Á \& Richart Martínez M. Enfermedad de Crohn, cronicidad y rol enfermero: revisión integradora y estado de la cuestión. Revista Electrónica Trimestral de Enfermería: Enfermería Global 2014 [citado el 26 de Septiembre de 2015]; Octubre (36) 323-335. Disponible en: http://scielo.isciii.es/pdf/eg/v13n36/revision3.pdf

7. Swendeman D, Ingram BL, \& Rotheram-Borus MJ. Common elements in selfmanagement of HIV and other chronic illnesses: an integrative framework. AIDS Care 2009; 21(10), 1321-1334. doi:10.1080/09540120902803158

8. Teesson M, Mitchell PB, Deady M, Memedovic S, Slade T, \& Baillie A. Affective and anxiety disorders and their relationship with chronic physical conditions in Australia: findings of the 2007 National Survey of Mental Health and Wellbeing. Australian and New Zealand Journal of Psychiatry 2011; 45(11), 939-946. doi:10.3109/00048674.2011.614590

9. González E, Elorza J \& Failde I. Comorbilidad psiquiátrica y fibromialgia. Su efecto sobre la calidad de vida de los pacientes. Actas Espanolas de Psiquiatria 2010; 38(5).

Disponible en: http://search.ebscohost.com/login.aspx?direct=true\&profile=ehost\&scope=site\&aut 
htype $=$ crawler \&jrnl=11399287\&AN=56631477\&h=GM6R5eT2vW1K0GKUIcXt37G qhiydJa\%2BgBoeZQy9w6RcsPCE2\%2FBWs4VKo0FMaSzQJrM8yvveXzXNfvluJL V2cGA\%3D\%3D\&crl=c

10. Lim L, Jin A-Z, \& Ng T-P. Anxiety and depression, chronic physical conditions, and quality of life in an urban population sample study. Social Psychiatry and Psychiatric Epidemiology 2012; 47(7), 1047-1053. doi:10.1007/s00127-011-0420-6

11. Egede LE. Major depression in individuals with chronic medical disorders: prevalence, correlates and association with health resource utilization, lost productivity and functional disability. Gen Hosp Psychiatry 2007; 29: 409-416

12. Edwards RR., Calahan C, Mensing G, Smith M, \& Haythornthwaite JA. Pain, catastrophizing, and depression in the rheumatic diseases. Nature Reviews Rheumatology, 2011; 7(4), 216-224. doi:10.1038/nrrheum.2011.2

13. Kristofferzon M-L, Lindqvist R \& Nilsson A. Relationships between coping, coping resources and quality of life in patients with chronic illness: a pilot study: Chronic illness relations between coping and QoL. Scandinavian Journal of Caring Sciences 2011 ; 25(3), 476-483. doi:10.1111/j.1471-6712.2010.00851.x

14.Büssing A, Ostermann T, Neugebauer EA, \& Heusser P. Adaptive coping strategies in patients with chronic pain conditions and their interpretation of disease. BMC Public Health 2010; 10(1), 507

15. Winters CA, Cudney S \& Sullivan T. Expressions of depression in rural women with chronic illness. Rural \& Remote Health 2010; 10(4). Disponible en: http://search.ebscohost.com/login.aspx?direct=true\&profile=ehost\&scope=site\&aut htype=crawler\&jrnl=14456354\&AN=57827873\&h=HmuQpIMNemQkOfu6iCkjVTBp Eq4CeHQ93hVbY4C\%2F0gbp6pMKSOvKV4KfjD4Gn8xSqjYdY8BOq1dCxl5xxe5 $\% 2 \mathrm{~B} 8 \mathrm{~A} \% 3 \mathrm{D} \% 3 \mathrm{D} \& \mathrm{crl}=\mathrm{C}$

16. Beck AT, Ward $\mathrm{CH}$, Mendelson M, Mock J \& Erbaugh J. An inventory for measuring depression. Archives of General Psychiatry 1961; 4, 561-571.

17.Spielberger CD, Gorsuch RL \& Lushene RE. STAl. Cuestionario de Ansiedad Estado/Rasgo. Madrid: TEA Ediciones 1999. (Trabajo original publicado en 1970)

18. Alonso J, Prieto L, Antó JM. La versión española del SF-36 HealthSurvey (Cuestionario de salud SF36): un instrumento para la medida de los resultados clínicos. MedClin (Barc) 1995; 104: 771-776.

19.Lorig, K. R., Ritter, P.L., Gonzalez, V.M Hispanic chronic disease selfmanagement: a randomized community-based outcome trial. Nurs Res 2003, 52: 361-9.

20. Cano FJ, Rodríguez Franco L \& García J. Adaptación española del Inventario de Estrategias de Afrontamiento. Actas Esp Psiquiatr 2007; 35(1), 29-39.

21. Soriano J \& Monsalve V. CAD: cuestionario de afrontamiento ante el dolor crónico. Rev Soc Esp Dolor 2002, 9(1), 13-22.

22. Rodríguez L, Cano FJ, Blanco A. Evaluación de las estrategias de afrontamiento del dolor crónico. Actas Españolas de Psiquiatría 2004, 32(2), 82-91.

23. Alonso J, Regidor E, Barrio G, Prieto L, Rodríguez C, De la Fuente L. Valores poblacionales de referencia de la versión española del Cuestionario de Salud SF36. Med Clin (Barc) 1998; 111: 410-416.

24. Hervás A., Zabaleta A., Miguel G, Beldarrain O, \& Díez, J. Calidad de vida relacionada con la salud en pacientes con diabetes mellitus tipo 2. Anales del sistema sanitario de Navarra; 2008. 30, 45-52.

25. Ku DY, Park YS, Chang HJ, Kim SR, Ryu JW \& Kim WJ. Depression and Life Quality in Chronic Renal Failure Patients with Polyneuropathy on Hemodialysis. Annals of Rehabilitation Medicine, 2012; 36(5), 702. doi:10.5535/arm.2012.36.5.702 
26. Kil SY, Oh WO, Koo BJ \& Suk MH. Relationship between depression and healthrelated quality of life in older Korean patients with chronic obstructive pulmonary disease. Journal of Clinical Nursing, 2010; 19(9-10), 1307-1314. doi:10.1111/j.1365-2702.2009.03053.x

27. Theofilou, P. Depression and Anxiety in Patients with Chronic Renal Failure: The Effect of Sociodemographic Characteristics. International Journal of Nephrology 2011; 1-6. doi:10.4061/2011/514070

28. Soucase B, Monsalve V \& Soriano JF. Afrontamiento del dolor crónico: el papel de las variables de valoración y estrategias de afrontamiento en la predicción de la ansiedad y la depresión en una muestra de pacientes con dolor crónico. Rev Soc Esp Dolor 2005; 12, 8-16.

29. Almeida GC \& Rivas V. Estrategias de afrontamiento en adultos con diabetes mellitus Tipo 2.2 Disponible en: http://www.archivos.ujat.mx/dip/divulgacion\%20y\%20video\%20cinetifico\%202008/ DACS/VRivasA\%202.pdf

30. Vilhena E, Pais-Ribeiro J, Silva I, Pedro L, Meneses RF, Cardoso H, da Silva A, Mendonça D. Psychosocial factors as predictors of quality of life in chronic portuguese patients. Health and Quality of Life Outcomes 2014, 12(1), 3.

Recibido: 16 de noviembre 2015;

Aceptado: 22 de enero 2016 